\title{
Identification of pterygium-related long non-coding RNAs and expression profiling by microarray analysis
}

\author{
JIN LIU $^{1 *}$, XIANGYA DING ${ }^{2 *}$, LI YUAN $^{1}$ and XIAOJUN ZHANG ${ }^{1}$ \\ ${ }^{1}$ Department of Ophthalmology, The Second Affiliated Hospital of Nanjing Medical University, Nanjing, Jiangsu 210003; \\ ${ }^{2}$ Department of Microbiology, Nanjing Medical University, Nanjing, Jiangsu 210029, P.R. China
}

Received January 27, 2016; Accepted June 2, 2016

DOI: $10.3892 /$ ijmm.2016.2641

\begin{abstract}
Pterygium is a common degenerative and proliferative disease of the ocular surface. It becomes a significant sight-threatening complication once its ingrowth covers the pupil. The proliferative capacities of pterygial cells give pterygia the appearance of having a mechanism similar to tumorigenesis. Long non-coding RNAs (IncRNAs) are key regulators of gene expression. The expression levels of certain lncRNAs are associated with a number of diseases, such as different tumors and metabolic disorders, among others. However, the contributions of lncRNAs to pterygium remain largely unexplored. In this study, we constructed pterygium-related lncRNA libraries using microarray analysis to investigate the potential roles of lncRNAs in the development of pterygium. A total of 3,066 upregulated and 1,646 downregulated lncRNAs were identified in pterygium tissues compared with paired adjacent normal conjunctival tissues (log fold change $>2.0$ ). Quantitative polymerase chain reaction (qPCR) was performed to validate 3 upregulated and 2 downregulated lncRNAs in 10 patients. Bioinformatics analyses (Gene Ontology analysis and pathway analysis) were used for further research. Pathway analysis indicated that 82 pathways corresponded to the downregulated transcripts and that 15 pathways corresponded to the upregulated transcripts ( $p$-value cut-off, 0.05). Our results reveal differentially expressed lncRNAs in pterygium and suggest that lncRNAs may be the novel molecular targets for the treatment of pterygium.
\end{abstract}

\section{Introduction}

Pterygium is a common benign fibrovascular lesion of the ocular surface in which the wedge-shaped ingrowth of conjunctival tissue invades the peripheral cornea (1). It becomes a significant

Correspondence to: Dr Xiaojun Zhang, Department of Ophthalmology, The Second Affiliated Hospital of Nanjing Medical University, 262 Zhongshan North Road, Gulou, Nanjing, Jiangsu 210003, P.R. China E-mail: zhangxiaojun@njmu.edu.cn

*Contributed equally

Key words: pterygium, long non-coding RNA, PISRT1, microarray, proliferation sight-threatening complication once the ingrowth covers the pupil. Surgical excision can be a useful therapy for pterygia; however, recurrences are common. Thus, there is a significant need to gain more insight into pterygium formation and recurrence, in order to enable the design of novel therapeutic strategies, either for inhibiting pterygium growth, regressing pterygia or preventing recurrence (2). Recent studies provide evidence that pterygium is a stem cell disorder with premalignant characteristics $(3,4)$, and that epithelial-mesenchymal transition (EMT) may play a key role in the pathogenesis of this disease $(5,6)$. Although considerable progress has been made towards understanding the etiology of the disease, the pathogenesis of pterygium is not yet completely understood $(7,8)$.

Long non-coding RNAs (lncRNAs) are recognized as being longer than 200 nucleotides in length without protein coding properties (9). They can be classified into 6 categories according to their genomic proximity to protein-coding genes: exon sense-overlapping, intron sense-overlapping, intronic antisense, natural antisense, bidirectional and intergenic lncRNAs (10). Recently, lncRNAs have been implicated in regulating a number of biological processes, such as gene imprinting, dosage compensation, cell cycle regulation, cell pluripotency, meiotic entry and telomere length $(10,11)$. lncRNAs regulate these processes at the transcriptional, post-transcriptional and epigenetic levels $(12,13)$. Moreover, dysregulated lncRNAs have been linked to the states of different diseases, such as cancer development and neurological disorders. Pterygium is a complex pathological process mediated by the dysfunction of the gene regulatory network; however, there is little data available on lncRNAs in pterygium. The features of lncRNAs expression and their potential biological function in pterygium require further investigation.

We hypothesized that lncRNAs may be involved in the molecular pathogenesis of pterygium. To examine this hypothesis, in this study, the global expression profiles of lncRNAs were analyzed by microarray analysis and quantitative polymerase chain reaction (qPCR) in human pterygium samples compared to adjacent normal conjunctival samples. Our results are likely to provide important insight into the pathogenesis of pterygium.

\section{Patients and methods}

Patient sample preparation. A total of 12 pterygium specimens were obtained during pterygium surgery at the Second 
Affiliated Hospital of Nanjing Medical University. The patients were in good health and their age ranged from 49 to 79 years, with 5 males and 7 females (Table I). All patients underwent conventional excision of pterygium with auto-transplantation of the conjunctiva. In the same patient, $1.5 \times 1.5 \mathrm{~mm}$ conjunctival tissues were obtained from the inferior temporal quadrant of the bulbar conjunctiva during surgery. Clinical details pertaining to the study patients are described in Table I. The study protocol was approved by the Ethics Review Committee of the Second Affiliated Hospital of Nanjing Medical University, Nanjing, China and all participants gave informed consent to the treating surgeon. All tissues were immediately frozen in liquid nitrogen and stored in a freezer at $-80^{\circ} \mathrm{C}$ until use.

RNA extraction and quality control. To extract RNA from tissues, we resuspended the frozen tissues in TRIzol reagent (Life Technologies, Carlsbad, CA, USA) and finally eluted into $50 \mu \mathrm{l}$ of elution solution according to the manufacturer's instructions. RNA quantity and quality were measured using a NanoDrop ND-1000 spectrophotometer. RNA integrity was assessed by standard denaturing agarose gel electrophoresis. We stored all the RNA samples at $-80^{\circ} \mathrm{C}$ until further processing. Complementary DNA (cDNA) was synthesized from $1 \mu \mathrm{g}$ of total RNA using a PrimesScript ${ }^{\mathrm{TM}}$ RT Master Mix kit (Takara, Dalian, China) with random hexamer primers in a final volume of $20 \mu \mathrm{l}$. The reverse transcription reactions were incubated at $37^{\circ} \mathrm{C}$ for $15 \mathrm{~min}, 85^{\circ} \mathrm{C}$ for $5 \mathrm{sec}$ and $4^{\circ} \mathrm{C}$ for $10 \mathrm{~min}$.

Microarray analysis. Arraystar Human LncRNA Microarray V3.0 is designed for the global profiling of human lncRNAs and protein-coding transcripts, which is updated from the previous Microarray V2.0. Approximately 30,586 lncRNAs and 26,109 coding transcripts can be detected by the thirdgeneration lncRNA microarray. The lncRNAs were obtained from authoritative databases (RefSeq, Ensembl and UCSC knowngenes, and others). The mRNAs were collected from RefSeq and GENCODE.

RNA labeling and array hybridization. RNA labeling and array hybridization were performed according to the Agilent One-Color Microarray-Based Gene Expression Analysis protocol (Agilent Technologies, Santa Clara, CA, USA) with minor modifications. Briefly, mRNA was purified from total RNA following the removal of rRNA (mRNA-ONLY ${ }^{\mathrm{TM}}$ Eukaryotic mRNA Isolation kit; Epicentre, Madison, WI, USA). Each sample was then amplified and transcribed into fluorescent cRNA along the entire length of the transcripts without 3 ' bias utilizing a mixture of oligo(dT) and random primers (Arraystar Flash RNA Labeling kit; Arraystar, Rockville, MD, USA). The labeled cRNAs were purified using an RNeasy Mini kit (Qiagen, Hilden, Germany). The concentration and specific activity of the labeled cRNAs (pmol Cy3/ $\mu \mathrm{g}$ cRNA) were measured using a NanoDrop ND-1000 spectrophotometer. A total of $1 \mu \mathrm{g}$ of each labeled cRNA was fragmented by the addition of $5 \mu \mathrm{l} 10 \mathrm{X}$ blocking agent and $1 \mu \mathrm{l}$ of $25 \mathrm{X}$ fragmentation buffer, then the mixture was heated at $60^{\circ} \mathrm{C}$ for $30 \mathrm{~min}$. Finally, $25 \mu 12 \mathrm{X}$ GE hybridization buffer were added to dilute the labeled cRNA. Subsequently, $50 \mu 1$ of hybridization solution were dispensed into the gasket slide and assembled to the IncRNA expression microarray slide. The slides were incubated for $17 \mathrm{~h}$ at $65^{\circ} \mathrm{C}$ in an Agilent Hybridization Oven. The hybridized arrays were washed, fixed and scanned with using the Agilent DNA Microarray Scanner (part no. G2505C; Agilent Technologies).

$q P C R$. The selected lncRNAs and the primers (Table II) used for qPCR were designed and synthesized by Generay Biotech (Shanghai, China). $\beta$-actin was used as an internal control for the tissue samples. qPCR was performed using the ViiA 7 Real-time PCR system (Applied Biosystems, Foster City, CA, USA) with the SYBR expression assay system (Takara). The PCR reaction conditions were as follows: an initial denaturation at $95^{\circ} \mathrm{C}$ for $10 \mathrm{~min}$, followed by $40^{*} \mathrm{PCR}$ cycles at $95^{\circ} \mathrm{C}$ for $10 \mathrm{sec}$ and $60^{\circ} \mathrm{C}$ for $60 \mathrm{sec}$. Finally annealing and extension at $95^{\circ} \mathrm{C}$ for $10 \mathrm{sec}, 60^{\circ} \mathrm{C}$ for $60 \mathrm{sec}$ and $95^{\circ} \mathrm{C}$ for $15 \mathrm{sec}$. Each sample was assayed in triplicate. We used the standard curve method to determine the fold change in gene expression in the pterygium samples relative to the adjacent normal conjunctival samples.

Gene Ontology $(G O)$ enrichment and pathway analysis. The GO project (http://www.geneontology.org) provides a controlled vocabulary to describe gene and gene product attributes (14). GO categories were considered as significantly enriched only if the Fisher's exact probability (P-value) was $<0.05$. Pathway analysis is a functional analysis mapping genes to KEGG pathways (15). The P-value (EASE-score, Fisher P-value or hypergeometric P-value) denotes the significance of the pathway correlated to the conditions. The lower the P-value, the more significant the pathway.

Statistical analysis. Agilent Feature Extraction software (version 11.0.1.1; Agilent Technologies) was used to analyze acquired array images. Quantile normalization and subsequent data processing were performed with using the GeneSpring GX v12.1 software package (Agilent Technologies). Following quantile normalization of the raw data, IncRNAs and mRNAs that at least 2 out of 4 samples have flags in Present or Marginal ('All Targets Value') were selected for further data analysis. Differentially expressed lncRNAs and mRNAs with statistical significance between the 2 groups were identified through P-value/FDR filtering and fold change filtering. Hierarchical clustering and combined analysis were performed using homemade scripts.

The data were analyzed using the SPSS 20.0 software package (SPSS Inc., Chicago, IL, USA). Differential expression levels of lncRNAs were compared by an independent-samples t-test between 2 groups. Fisher's exact test was used in GO and pathway analyses. All values are expressed as the means + SEM. All experiments were repeated at least 3 times. A value of $\mathrm{P}<0.05$ was considered to indicate a statistically significant difference.

\section{Results}

Profile of microarray data. According to microarray expression profiling data, 25,770 $\mathrm{lncRNAs}$ were detected. In addition, 4,712 lncRNAs (log fold change $>2.0$ ) were found to be differentially expressed between the pterygium samples and the paired adjacent normal conjunctival tissue samples (Table III and IV). 
Table I. Clinical and demographic information for patients.

\begin{tabular}{cccl}
\hline Case no. & Age & Gender & Case details \\
\hline 1 & 58 & M & Primary \\
2 & 63 & F & Primary \\
3 & 49 & M & Primary \\
4 & 72 & M & Recurrent \\
5 & 50 & F & Primary \\
6 & 66 & F & Primary \\
7 & 79 & M & Primary \\
8 & 68 & F & Primary \\
9 & 61 & F & Primary \\
10 & 70 & M & Primary \\
11 & 54 & F & Primary \\
12 & 72 & F & Primary \\
\hline
\end{tabular}

Table II. Primers used for PCR in this study.

\begin{tabular}{llc}
\hline \multirow{2}{*}{ lncRNAs } & \multicolumn{1}{c}{ Primers } & $\begin{array}{c}\text { Length } \\
\text { (bp) }\end{array}$ \\
\hline PISRT1 & F: 5'-GATCCAGGCTCTTGACCACTC-3' & \\
& R:5'-CGGCTTCTCTCTGGAACTCAG-3' & 88 \\
LOC283761 & F: 5'-AAGACCCTCCCTTGAACCGT-3' & \\
& R:5'-TCAAACCCTGTGGGGCTTTC-3' & 153 \\
FOXD2-AS1 & F: 5'-AGTGGGGAATGAGGATGGGT-3' & \\
& R:5'-CGGCGTGTAATTGGTAGGAG-3' & 142 \\
LPAL2 & F: 5'-ACGCCACACCAGCACAGTAG-3' & \\
& R:5'-GCACGAACACAGTCCCTTCAT-3' & 178 \\
SNHG1 & F: 5'-ATGAAACAGCAGTTGAGGGT-3' & \\
& R:5'-AAAAACAAAAGGGCAGGTAG-3' & 102 \\
3 -actin & F: 5'-GTGGCCGAGGACTTTGATTG-3' & \\
& R:5'-CCTGTAACAACGCATCTCATATT-3' & 73 \\
\hline
\end{tabular}

lncRNAs, long non-coding RNAs.

All these lncRNAs were obtained from authoritative databases,such as RefSeq, UCSC_Knowngenes, Ensembl and other related databases (Fig. 1A). Hierarchical Cluster analysis revealed IncRNA expression patterns (Fig. 1B). A scatter plot was used for assessing variations in lncRNA expression between the 2 groups of pterygium samples and adjacent normal conjunctival samples (Fig. 1C). A total of 3,066 upregulated and 1,646 downregulated IncRNAs were identified in the pterygium tissues compared with the paired adjacent normal conjunctival tissues. A volcano plot filtering between the 2 groups initially revealed differentially expressed lncRNAs with statistical significance (fold change $>2.0, \mathrm{P}<0.05$ ) (Fig. 1D).

Confirmation by $q P C R$. By using $\mathrm{q} P C R, 3$ upregulated $\operatorname{lncRNAs}$ [polled intersex syndrome regulated transcript 1 (PISRT1), LOC283761 and FOXD2 antisense RNA 1 (FOXD2-AS1)] and 2 downregulated lncRNAs [lipoprotein, Lp(A)-like 2, pseudogene (LPAL2) and small nucleolar RNA host gene 1 (SNHG1)] with log fold changes $>10$ were randomly selected to test and
Table III. Numbers of significantly differentially expressed lncRNAs.

\begin{tabular}{|c|c|c|c|c|}
\hline & & $\begin{array}{c}\text { Fold change } \\
2-5\end{array}$ & $\begin{array}{c}\text { Fold change } \\
5-10\end{array}$ & $\begin{array}{l}\text { Fold change } \\
\qquad>10\end{array}$ \\
\hline \multirow[t]{2}{*}{$\operatorname{lncRNA}$} & Upregulation & 1308 & 943 & 815 \\
\hline & Downregulation & 827 & 554 & 265 \\
\hline
\end{tabular}

lncRNAs, long non-coding RNAs.

Table IV. Top 10 significantly differently expressed lncRNAs.

\begin{tabular}{llcc}
\hline Seqname & GeneSymbol & Regulation & Fold-change \\
\hline NR_026878 & FOXD2-AS1 & Up & 92.676596 \\
ENST00000580781 & RP11-78F17.1 & Up & 88.7604945 \\
ENST00000530941 & RP11-702F3.4 & Up & 82.0072128 \\
ENST00000423020 & RP5-963E22.4 & Up & 75.080159 \\
ENST00000549419 & RP11-611E13.2 & Up & 70.6282429 \\
ENST00000445586 & AF196972.9 & Up & 66.0127502 \\
ENST00000563678 & KIAA0664L3 & Up & 63.4678339 \\
NR_027074 & LOC283761 & Up & 61.2789639 \\
ENST00000441203 & RP3-416J7.2 & Up & 58.0589993 \\
NR_024564 & LOC100130264 & Up & 56.0539117 \\
NR_024396 & LINC00638 & Down & 113.4657051 \\
ENST00000510017 & WI2-2373I1.2 & Down & 104.5215055 \\
NR_024526 & ARL6IP6 & Down & 89.9350702 \\
NR_024602 & CLCA4 & Down & 68.6207695 \\
NR_038103 & TECR & Down & 57.4518562 \\
ENST00000554583 & RP11-398J10.2 & Down & 54.8515259 \\
ENST00000493239 & RP11-61L23.2 & Down & 48.1050017 \\
ENST00000505133 & RP11-420A23.1 & Down & 46.1669142 \\
ENST00000562356 & RP11-217B1.2 & Down & 43.4260703 \\
ENST00000430751 & RP3-434O14.8 & Down & 38.9352585 \\
& & &
\end{tabular}

lncRNAs, long non-coding RNAs.

verify the microarray data in 10 different samples of pterygium tissues and paired adjacent normal conjunctival tissues (Fig. 2). The results of qPCR and microarray data analysis were consistent. Thus, microarray data profiling indicated a series of IncRNAs which were constantly differentially expressed between the pterygium tissues and the paired adjacent normal conjunctival tissues.

Expression signatures of deregulated lncRNAs between pterygium tissues and paired adjacent normal conjunctival tissues. As IncRNA expression was tissue-specific, to further study the lncRNA expression patterns in pterygium tissues, we first investigated general signatures of deregulated lncRNAs which log fold changes $>2.0$, including lncRNA classification, length distribution and chromosome distribution. According to the IncRNA position on the genome, we attributed lncRNA into 6 categories (bidirectional, exon sense-overlapping, intergenic, intronic antisense and natural antisense). Classification analysis revealed that the majority of the IncRNAs in our microarray belong to intergenic IncRNAs (Fig. 3A) and are distributed 

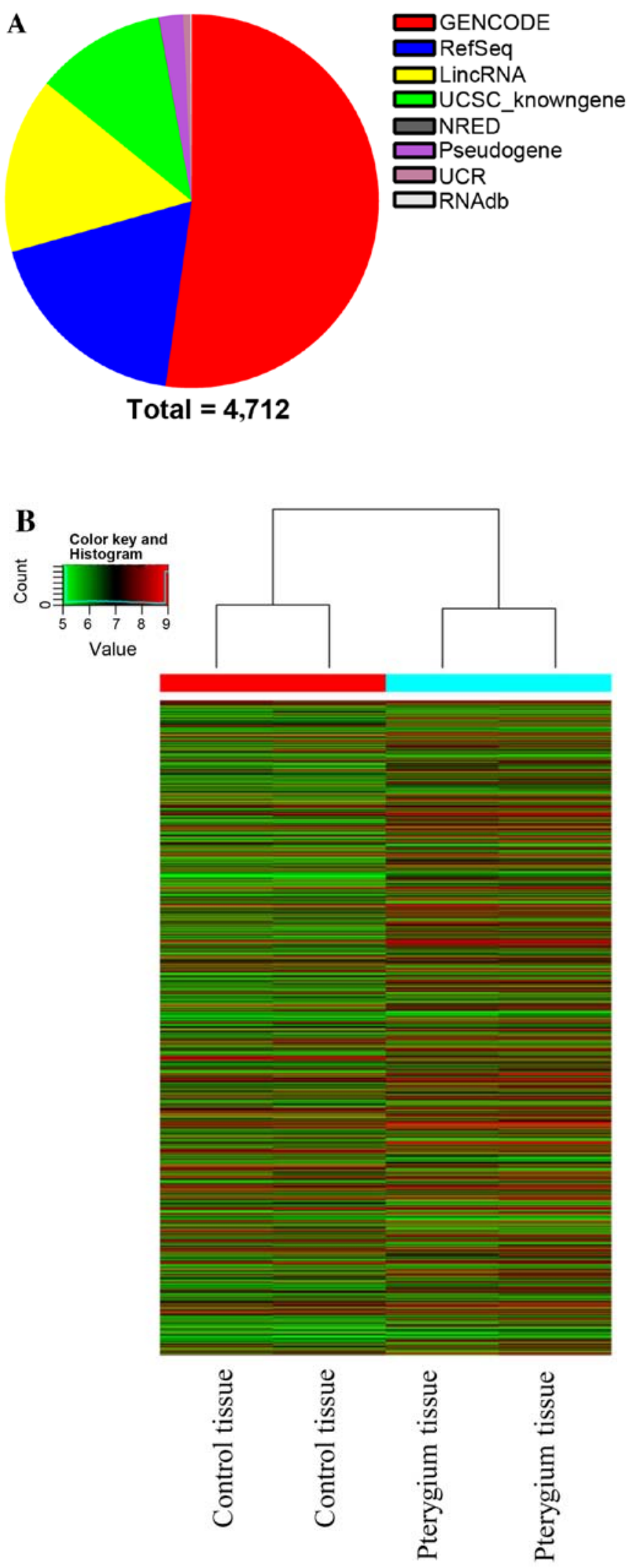
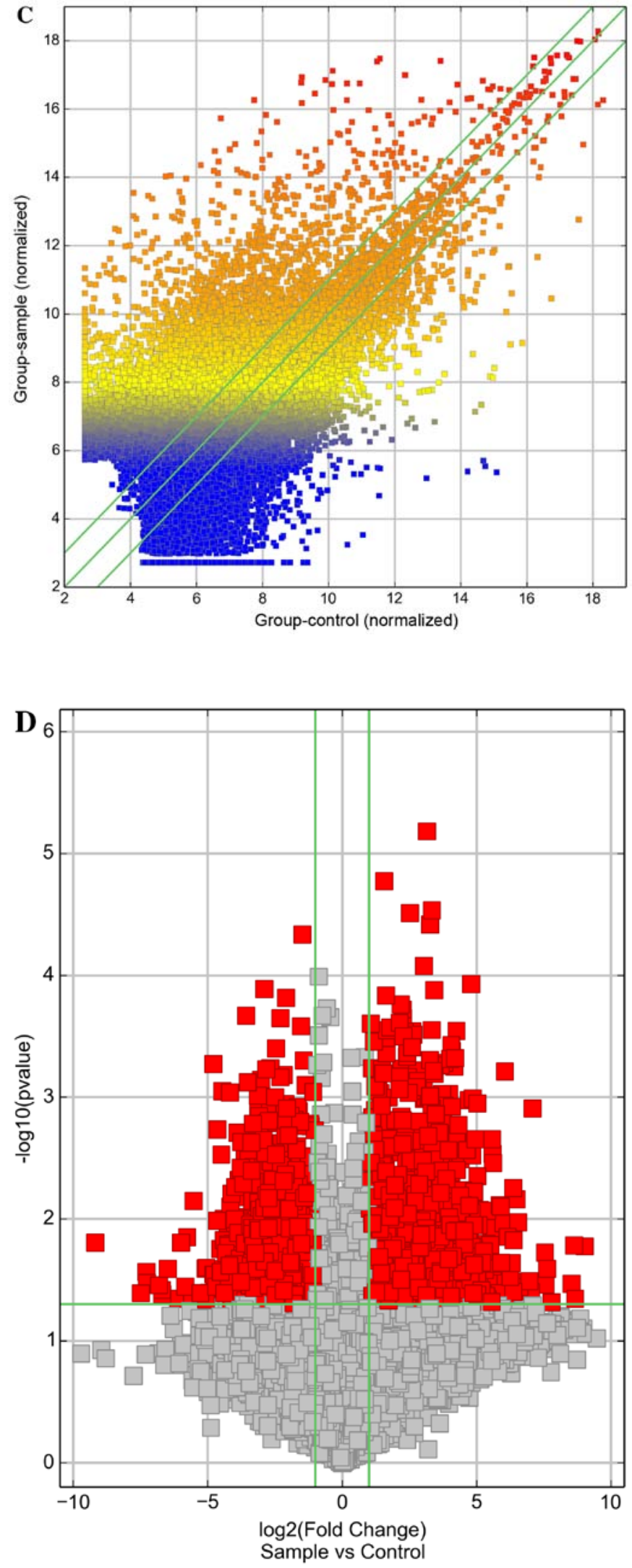

Figure 1. The differentially expressed profling in pterygium was compared with paired adjacent normal conjunctival tissues. (A) Using second-generation long non-coding RNAs (lncRNA) microarray, 4,712 lncRNAs (log fold-changes $>2.0$ ) were detected. The pie chart shows the most authoritative databases. (B) Differentially expressed lncRNAs in pterygium and paired adjacent normal conjunctival tissues were analyzed using hierarchical clustering. Hierarchical clustering analysis arranges samples into groups by expression level: 'red' indicates high relative expression, and 'green' indicates low relative expression. (C) The scatter plot is a visualization method used for assessing variations in lncRNA expression between pterygium and paired adjacent normal conjunctival tissues. The values of the $\mathrm{x}$ - and $\mathrm{y}$-axis in the scatter plot are the averaged normalized signal values of the group $(\log 2$ scaled). The green lines are fold change lines. (D) Volcano plot analysis of lncRNA microarray data of differentially expressed lncRNAs between pterygium and paired adjacent normal conjunctival tissues. The red points in the plot represent the differentially expressed lncRNAs with statistical significance (fold change $>2.0$, $\mathrm{P}<0.05$ ), while the vertical lines correspond to 2.0 -fold up- and downregulation, and the horizontal line is $\mathrm{P}=0.05$. 

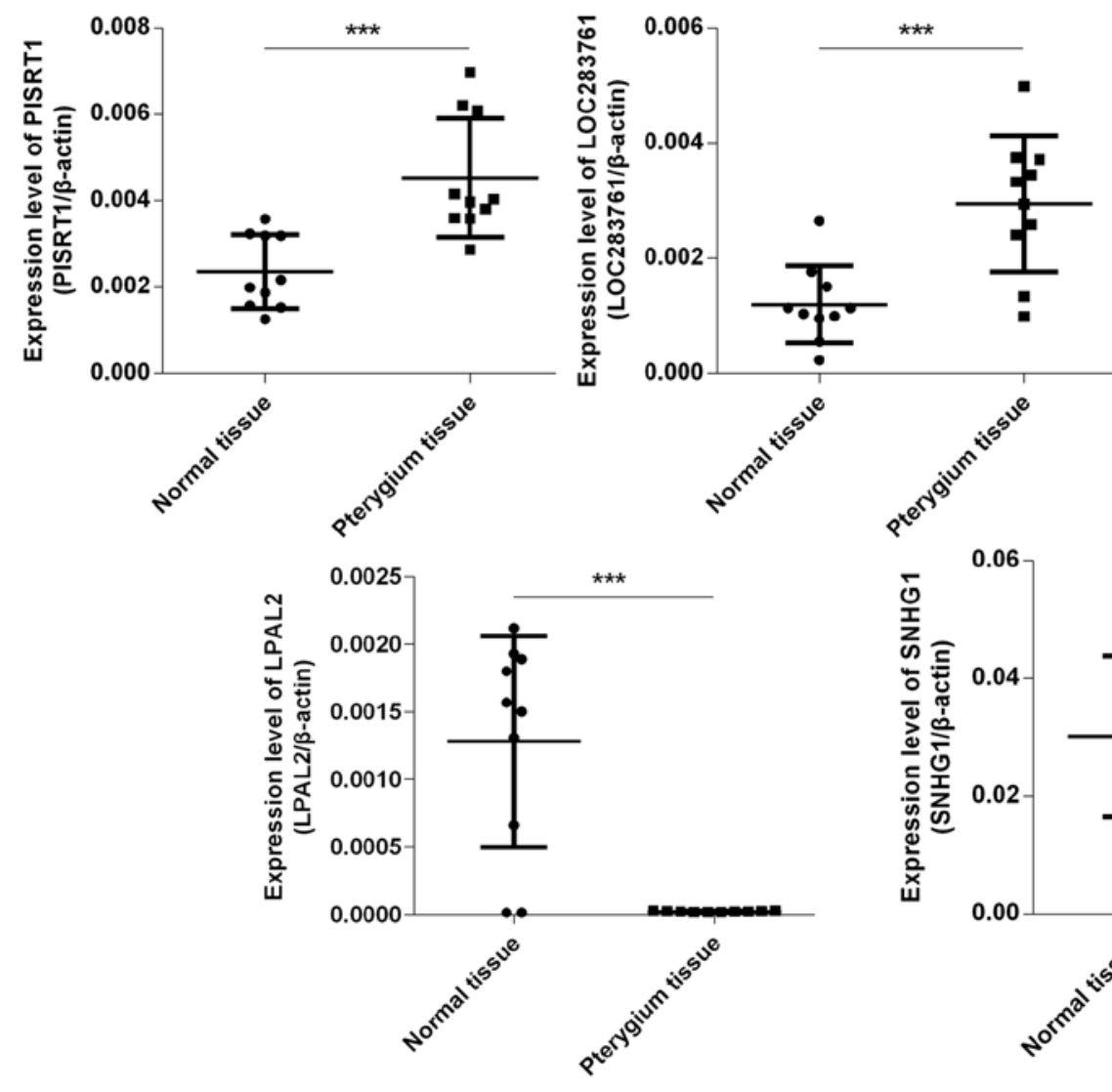

Figure 2. Differential expression of long non-coding RNAs (lncRNAs) between pterygium and paired adjacent normal conjunctival tissues were validated by qPCR. ${ }^{* *} \mathrm{P}<0.01$ and ${ }^{* * *} \mathrm{P}<0.001$.

401-800 nt in length (Fig. 3B). Chromosome distribution shows that upregulated and downregulated lncRNAs have different chromosomal locations (Fig. 3C and D). For the downregulated lncRNAs on the Y chromosome, all 125 transcripts were antisense, indicating that the mechanisms of the $\mathrm{Y}$ chromosome involved in pterygium formation may differ from those of other chromosomes.

GO analysis. GO analysis was performed to determine the gene and gene product enrichment, which covered 3 domains: biological processes, cellular components and molecular functions. Fisher's exact test was used to determine whether the overlap between the differentially expressed gene list and the GO annotation list was greater than that expected by chance (a p-value $<0.05$ is recommended). We found that the highest GO classification targeted by over-regulated transcripts were single-organism process (ontology:biological process), membrane (ontology:cellular component) and protein binding (ontology:molecular function). Furthermore, the highest GO classification targeted by the downregulated transcripts were cellular process (ontology:biological process), cell part (ontology:cellular component) and binding (ontology:molecular function). To analyze the candidate biological process of IncRNAs, we enriched the biological process of both the up- and downregulated lncRNAs. It is interesting that most biological processes of upregulated lncRNAs are involved in proliferation and differentiation, such as muticellular organismal process, single-multicellular organism process, cellular developmental process, cell differentiation, anatomical structure development, anatomical structure morphogenesis, single-organism developmental process, developmental process, organ development and system development, which shows that upregulated lncRNAs in pterygium tissues have a tight association with proliferation and differentiation (Fig. 4A). To the best of our knowlege, we are the first to report that pterygium-related lncRNAs are associated with proliferation and differentiation by microarray analysis. On the other hand, we found that the biological process of downregulated lncRNAs was enriched in cellular metabolic process, cellular macromolecule metabolic process and other metabolic processes (Fig. 4B).

Pathway analysis. We performed pathway analysis by mapping genes to KEGG pathways. Pathway analysis indicated that 82 pathways corresponded to downregulated transcripts and 15 pathways corresponded to upregulated transcripts. The top 10 pathways that were associated with upregulated and downregulated IncRNAs were both analyzed (Fig. 4C and D). The top pathways that were associated with the upregulated lncRNAs were enriched in the category 'hypertrophic cardiomyopathy', closely related with proliferation. Furthermore, the majority of the pathways that were associated with the downregulated lncRNAs were related to tumorigenesis, such as proteoglycans in cancer, colorectal cancer and prostate cancer. This analysis revealed that IncRNAs may play a key role in proliferation and may be involved in the pterygium developmental process.

As we found that upregulated lncRNAs may be associated with proliferation and the downregulated lncRNAs may influence pterygium development, we selected upregulated 

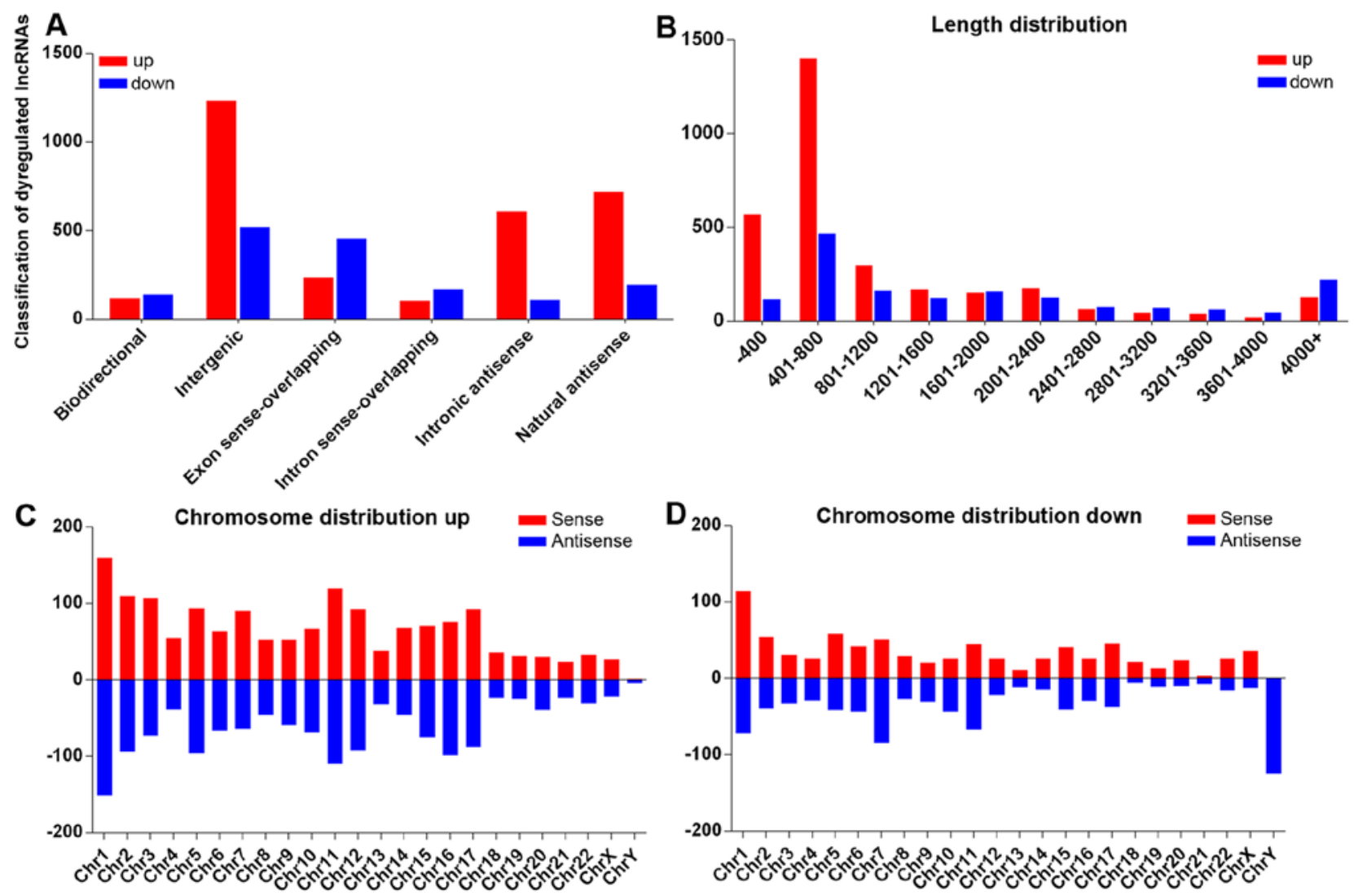

Figure 3. (A) Classification of deregulated long non-coding RNAs (lncRNAs). The lncRNAs are mainly intergenic. (B) Length distribution of the dysregulated lncRNAs. The lncRNAs are mainly between 400 and 800 bp in length. Chromosome distribution shows the numbers of (C) upregulated (up) and (D) downregulated (down) lncRNAs located on different chromosomes.

lncRNAs with a log fold change $>2.0$ in pterygium and the paired adjacent normal conjunctival tissues and their associated coding genes with a function of proliferation; we also selected downregulated lncRNAs with a log fold change $>2.0$ and their associated coding genes with a function of apoptosis.

\section{Discussion}

Pterygium has been considered a degenerative and proliferative process of the corneal limbus and is characterized by the invasion of a fleshy triangle of conjunctival tissue onto the cornea (16). The condition is associated with chronic ultraviolet radiation exposure and is characterized by the induction of cell proliferation, squamous metaplasia, goblet cell hyperplasia, inflammation, fibrosis, angiogenesis and extracellular matrix breakdown (8). Indeed, pterygium tissue shares the characteristics of many premalignant tissues, including epithelial proliferation, goblet cell hyperplasia, angiogenesis, inflammation and elastosis $(3,17)$. However, the mechanisms responsible for pterygium formation have not been fully clarified. This is critical to determine the expression profiles of lncRNAs in pterygium and to understand its pathogenesis.

Recent microarray analysis has demonstrated that microRNAs (miRNAs) and their targeted mRNAs, are functionally important in processes relevant to pterygium pathogenesis (1). The concerted upregulation of miR-221 is known to regulate downstream p27Kip1 gene expression (16), and evidence has indicated that the miR-200 family is a potential regulator of EMT in pterygium (1).

Mounting evidence has indicated that numerous lncRNAs play roles in multiple biological processes and regulate different diseases. Of all the functions of lncRNAs, the most important is their involvement in tumorigenesis, which has been shown in different cancer cells $(18,19)$. The proliferative capacities of pterygial cells have a mechanism similar to tumorigenesis. However, the roles of pterygium pathogenesis remain unclear.

In the present study, we investigated the IncRNA expression profiles of pterygium using microarray analysis and found that the IncRNA expression levels were altered compared to adjacent normal conjunctival tissues. From the microarray expression profiles, we found that 3,066 upregulated and 1,646 downregulated IncRNAs were significantly differentially expressed ( $>2.0$-fold) in all pterygium samples. Five lncRNAs were evaluated by qPCR to validate the consistency. The qPCR results and microarray data were consistent. Furthermore, we utilized GO analysis, pathway analysis and constructed a co-expression network to preliminarily examine the biological functions of these lncRNAs in the development of pterygium.

For pterygium, genetic mutations in development genes are common, as observed in other proliferative diseases, such as cancers. An over-regulated lncRNA, PISRT1, was found to be located near mitochondrial ribosomal protein S22 (MRPS22), which interacts with p53 and is considered a potential driver gene whose targets were shown to be involved in DNA repli- 
A

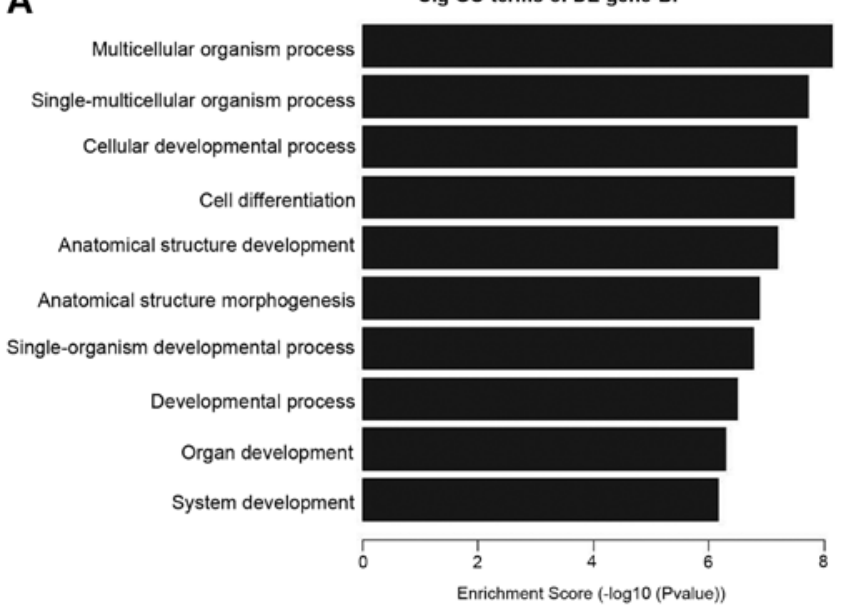

C

Hypertrophic cardiomyopathy (HCM) - Homo sapiens (human)
Cytokine-cytokine receptor interaction - Homo sapiens (human) Arhythmogenic right ventricular cardiomyopathy (ARVC) - Homo sapiens (human)

Glycosaminoglycan biosynthesis - keratan sulfate - Homo sapiens (human) Cardiac muscle contraction - Homo sapiens (human) Other types of O-glycan biosynthesis - Homo sapiens (human) Dilated cardiomyopathy - Homo sapiens (human) Collecting duct acid secretion - Homo sapiens (human) Bacterial invasion of epithelial cells - Homo sapiens (human) Tryptophan metabolism - Homo sapiens (human)
Sig GO terms of DE gene-BP

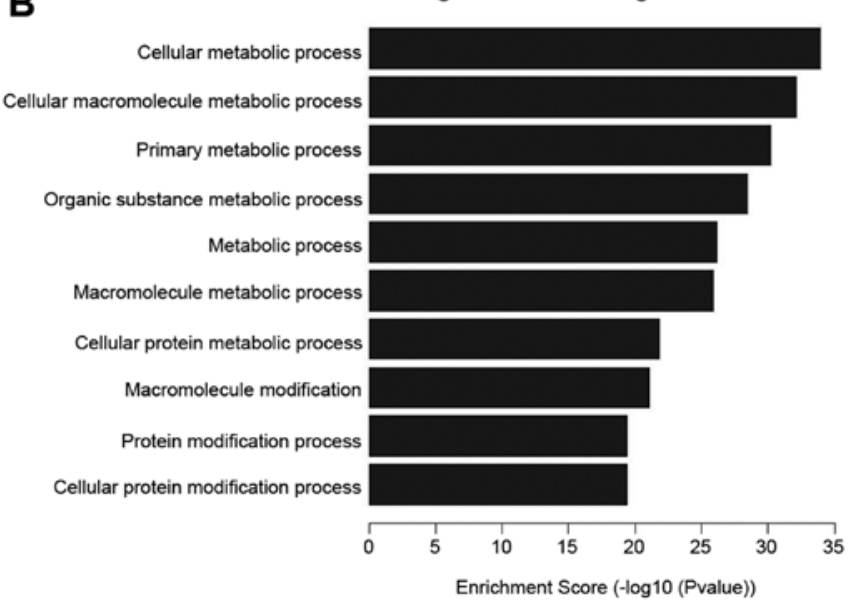

Sig pathway of DE gene

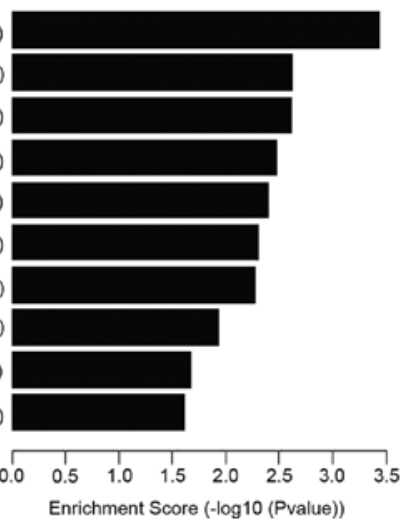

D

Sig pathway of DE gene

Protein processing in endoplasmic reticulum - Homo sapiens (human)

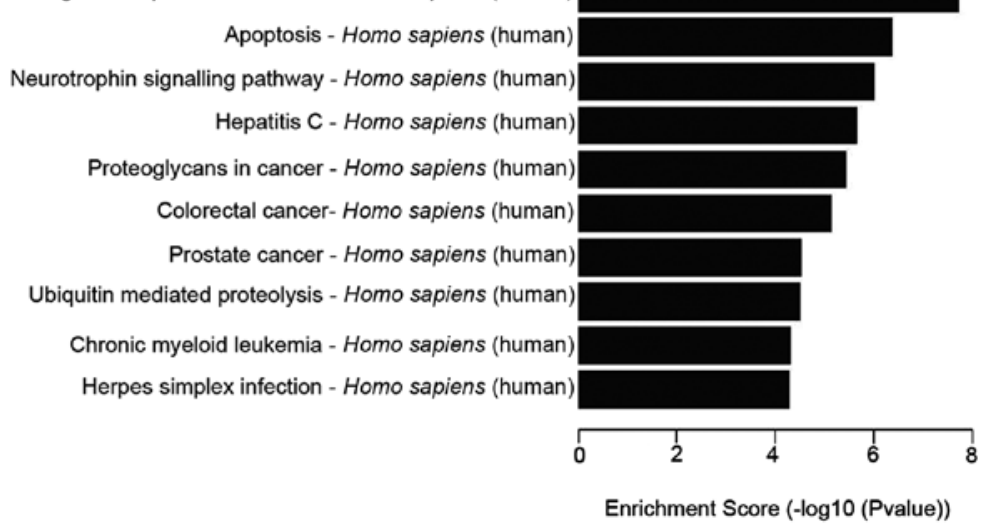

Figure 4. Gene Ontology (GO) and pathway analysis. The top $10 \mathrm{GO}$ terms that are associated with coding gene functions of (A) upregulated long non-coding RNAs (lncRNAs) and (B) downregulated lncRNAs are listed. The top 10 pathways that are associated with coding genes of (C) upregulated lncRNAs and (D) downregulated lncRNAs are listed.

cation, cell cycle, mismatch repair, p53 signaling pathway and other lung cancer related signaling pathways, and many immunological pathways (20). An intronic antisense relationship between the over-regulated lncRNA, PISRT1, and MRPS22 may help us learn more about the association between pterygium and lncRNAs at the transcriptional level.

To understand the functions of IncRNAs, we applied pathway analysis to study the differentially expressed IncRNAs and found that 82 pathways corresponded to the downregulated transcripts and 15 pathways corresponded to the upregulated transcripts. Chronic inflammation is a critical process involved in the development and progression of pterygium, including the promotion of angiogenesis $(7,21,22)$. Pterygium research has uncovered multiple pro-inflammatory genes that are activated in pterygium tissue, including the pro-inflammatory transcription factor, nuclear factor- $\mathrm{\kappa B}(\mathrm{NF}-\kappa \mathrm{B})(22)$, and several cytokines, including tumor necrosis factor- $\alpha$ (TNF- $\alpha$ ) (23). Some of those pathways include the NF- $\mathrm{BB}$ signaling pathway and TNF 
signaling pathway, which is consistent with previous research. Moreover, one of these pathways, the 'Focal adhesion' signaling pathway has shown to modulate tumor signatures (24). These pathways were associated with outcomes for pterygium. In this study, 97 pathways corresponding to differentially expressed transcripts were found, and some or all may be involved in the pathogenesis of pterygium.

In conclusion, to the best of our knowledge, in this study, we report for the first time that lncRNAs are differentially expressed in pterygium compared with paired adjacent normal conjunctival tissues. Some of the changes identified in this study suggest new directions in the formation of pterygium. In the future, we will expand the number of enrolled patients, and further studies are warranted applying immunofluorescence, proteomics or protein level quantification of the lncRNA targets in patients with pterygium staged according to the severity level. Through further investigation, these aberrant lncRNAs may be biomarkers, molecular targets and prognostic markers in the medical treatment of pterygium.

\section{Acknowlegements}

The authors wish to thank Dr Chun Lu for his technical assistance and critical review of this manuscript.

\section{References}

1. Engelsvold DH, Utheim TP, Olstad OK, Gonzalez P, Eidet JR, Lyberg T, Trøseid AM, Dartt DA and Raeder S: miRNA and mRNA expression profiling identifies members of the miR-200 family as potential regulators of epithelial-mesenchymal transition in pterygium. Exp Eye Res 115: 189-198, 2013.

2. Al-Swailem S, Xu Z, Wu L, Hartsock MJ, Yiu SC and Duh EJ: Induction of endothelial RAGE expression in pterygium. Mol Vis 20: 1740-1748, 2014.

3. Chui J, Coroneo MT, Tat LT, Crouch R, Wakefield D and Di Girolamo N: Ophthalmic pterygium: A stem cell disorder with premalignant features. Am J Pathol 178: 817-827, 2011.

4. Hirst LW, Axelsen RA and Schwab I: Pterygium and associated ocular surface squamous neoplasia. Arch Ophthalmol 127: 31-32, 2009.

5. Ando R, Kase S, Ohashi T, Dong Z, Fukuhara J, Kanda A, Murata M, Noda K, Kitaichi N and Ishida S: Tissue factor expression in human pterygium. Mol Vis 17: 63-69, 2011.

6. Kato N, Shimmura S, Kawakita T, Miyashita H, Ogawa Y, Yoshida S, Higa K, Okano H and Tsubota K: Beta-catenin activation and epithelial-mesenchymal transition in the pathogenesis of pterygium. Invest Ophthalmol Vis Sci 48: 1511-1517, 2007.

7. Bradley JC, Yang W, Bradley RH, Reid TW and Schwab IR: The science of pterygia. Br J Ophthalmol 94: 815-820, 2010
8. Chui J, Di Girolamo N, Wakefield D and Coroneo MT: The pathogenesis of pterygium: Current concepts and their therapeutic implications. Ocul Surf 6: 24-43, 2008.

9. Rinn JL and Chang HY: Genome regulation by long noncoding RNAs. Annu Rev Biochem 81: 145-166, 2012.

10. Ponting CP, Oliver PL and Reik W: Evolution and functions of long noncoding RNAs. Cell 136: 629-641, 2009.

11. Yan B, Wang ZH, Liu JY, Tao ZF, Li XM and Qin J: Long noncoding RNAs: Versatile players in biologcial processes and human disorders. Epigenomics 6: 375-379, 2014.

12. Hung T and Chang HY: Long noncoding RNA in genome regulation: Prospects and mechanisms. RNA Biol 7: 582-585, 2010.

13. Wang KC and Chang HY: Molecular mechanisms of long noncoding RNAs. Mol Cell 43: 904-914, 2011.

14. Huntley RP, Harris MA, Alam-Faruque Y, Blake JA, Carbon S, Dietze H, Dimmer EC, Foulger RE, Hill DP, Khodiyar VK, et al: A method for increasing expressivity of Gene Ontology annotations using a compositional approach. BMC Bioinformatics 15: $155,2014$.

15. Khatri P, Sirota M and Butte AJ: Ten years of pathway analysis: Current approaches and outstanding challenges. PLoS Comput Biol 8: e1002375, 2012.

16. Wu CW, Cheng YW, Hsu NY, Yeh KT, Tsai YY, Chiang CC, Wang WR and Tung JN: MiRNA-221 negatively regulated downstream p27Kip1 gene expression involvement in pterygium pathogenesis. Mol Vis 20: 1048-1056, 2014.

17. Tung JN, Chiang CC, Tsai YY, Chou YY, Yeh KT, Lee H and Cheng YW: CyclinD1 protein expressed in pterygia is associated with $\beta$-catenin protein localization. Mol Vis 16: 2733-2738, 2010.

18. Wang J, Liu X, Wu H, Ni P, Gu Z, Qiao Y, Chen N, Sun F and Fan Q: CREB upregulates long non-coding RNA, HULC expression through interaction with microRNA-372 in liver cancer. Nucleic Acids Res 38: 5366-5383, 2010

19. Huang JL, Ren TY, Cao SW, Zheng SH, Hu XM, Hu YW, Lin L, Chen J, Zheng L and Wang Q: HBx-related long non-coding RNA DBH-AS1 promotes cell proliferation and survival by activating MAPK signaling in hepatocellular carcinoma. Oncotarget 6: 33791-33804, 2015.

20. Lazar V, Suo C, Orear C, van den Oord J, Balogh Z, Guegan J, Job B, Meurice G, Ripoche H, Calza S, et al: Integrated molecular portrait of non-small cell lung cancers. BMC Med Genomics 6: 53, 2013.

21. Detorakis ET and Spandidos DA: Pathogenetic mechanisms and treatment options for ophthalmic pterygium: Trends and perspectives (Review). Int J Mol Med 23: 439-447, 2009.

22. Mauro J and Foster CS: Pterygia: Pathogenesis and the role of subconjunctival bevacizumab in treatment. Semin Ophthalmol 24: 130-134, 2009.

23. Hong S, Choi JY, Lee HK, Seong GJ, Seo KY, Kim EK and Byeon SH: Expression of neurotrophic factors in human primary pterygeal tissue and selective TNF-alpha-induced stimulation of ciliary neurotrophic factor in pterygeal fibroblasts. Exp Toxicol Pathol 60: 513-520, 2008.

24. O'Donoghue LE, Ptitsyn AA, Kamstock DA, Siebert J, Thomas RS and Duval DL: Expression profiling in canine osteosarcoma: Identification of biomarkers and pathways associated with outcome. BMC Cancer 10: 506, 2010. 